\title{
Successful treatment of a 14-year-old patient with intestinal malrotation with laparoscopic Ladd procedure: case report and literature review
}

\author{
Yuka Nakajima ${ }^{1}$, Hiroyuki Sakata ${ }^{1}$, Tomohiro Yamaguchi ${ }^{1}$, Norichika Yoshie ${ }^{1}$, Taihei Yamada ${ }^{1}$, Takaaki Osako ${ }^{1}$, \\ Mariko Terashima' ${ }^{1}$ Naomi Mambo' ${ }^{1}$ Ryuta Saka², Satoko Nose², Takashi Sasaki², Hiroomi Okuyama², \\ Atsunori Nakao ${ }^{1 *}$ and Joji Kotani ${ }^{1}$
}

\begin{abstract}
Midgut malrotation is an anomaly of intestinal rotation that occurs during fetal development and usually presents in the neonatal period. We present a rare case of malrotation in a 14-year-old patient who presented with cramping, generalized right abdominal pain, and vomiting for a duration of one day. A computed tomography abdominal scan and upper gastrointestinal contrast studies showed malrotation of the small bowel without volvulus. Laparoscopy revealed typical Ladd's bands and a distended flabby third and fourth duodenal portion extrinsically obstructing the misplaced duodeno-jejunal junction. The Ladd procedure, including widening of the mesenteric base and appendectomy, was performed. Symptoms completely resolved in a half-year follow up period. Patients with midgut malrotation may present with vague abdominal pain, intestinal obstruction, or intestinal ischemia. The laparoscopic Ladd procedure is feasible and safe, and it appears to be as effective as the standard open Ladd procedure in the diagnosis and treatment of teenage or adult patients with intestinal malrotation.
\end{abstract}

Keywords: Malrotation, Laparoscopic surgery, Ladd procedure, Acute abdomen, Teenager

\section{Introduction}

Midgut malrotation is a congenital anomaly of intestinal rotation presenting mainly in childhood, usually within the first month of life. Midgut malrotation refers to a failure in the counter-clockwise rotation of the midgut, which results in the misplacement of the duodeno-jejunal junction to the right midline, comprising non-rotation and incomplete rotation of the superior mesenteric artery. Malrotation is typically diagnosed in the first few months of life, and $90 \%$ of cases are diagnosed during the first year. However, older children and adolescents are likely to present with recurrent abdominal pain, intermittent obstructive symptoms, or failure to thrive due to intestinal obstruction or intestinal ischemia [1-4].

We present the case of a symptomatic 14-year-old patient complaining of abdominal pain found to have

\footnotetext{
* Correspondence: atsunorinakao@aol.com

'Department of Emergency, Disaster and Critical Care Medicine, Hyogo

College of Medicine, 1-1 Mukogawa, Nishinomiya, Hyogo 663 8501, Japan

Full list of author information is available at the end of the article
}

intestinal malrotation that was successfully treated with a laparoscopic Ladd procedure. In adults or older children, the diagnosis is mostly incidental, based on investigation carried out for unrelated symptoms. Indeed, most adult patients are asymptomatic and incidentally, malrotation is often discovered later in life during surgery for other conditions. We diagnosed congenital intestinal malrotation, which rarely occurs in adults or older children, by using several modalities such as barium studies, computed tomography, and laparoscopy. We describe the clinical and radiological data of this patient followed by a brief review of the literature. This case report serves to demonstrate the benefits of laparoscopic surgery for malrotation. Also, the present case reminds us that intestinal malrotation should be considered in the differential diagnosis of a wide variety of symptoms and should be treated promptly once the diagnosis has been confirmed. 


\section{Presentation of case}

A 14-year-old man presented to our emergency center with cramping and generalized abdominal pain. His abdominal pain began the previous night shortly after eating and recurred intermittently. Multiple presentations with similar symptoms during his teenage years had failed to identify the cause of his pain. He had no history of previous abdominal surgeries. He was on no medication at the time and denied alcohol or tobacco use. The patient also vomited on the day of presentation with vomitus containing biliary contents. On physical examination, the patient's vital signs were: pulse, 67 beats/minute; blood pressure, $121 / 61 \mathrm{mmHg}$; body temperature, $36.9^{\circ} \mathrm{C}$; and respiration rate, 15 breaths/minute. He was well-nourished and alert without cyanosis. His abdomen was not distended, but his bowel sounds were weak. He exhibited no peritoneal signs; however mild diffuse tenderness to deep palpation was noted. His white blood cell count was $10160 / \mathrm{mm}^{3}$. Serum biochemistry and liver function test results were within normal limits, except a $\mathrm{C}$-reactive protein level of $4.2 \mathrm{mg} / \mathrm{dl}$.

Chest radiography did not reveal any signs of perforation of a hollow viscus. Ultrasonography demonstrated a fluidfilled, distended, small gut loop. No free liquid was visible between the intestinal segments or in the pelvis. Axial contrast-enhanced computed tomography (CT) obtained through the mid-abdomen showed an inverted relationship between the superior mesenteric artery (SMA) and superior mesenteric vein (SMV). The SMV was positioned to the anterior of the SMA (Figure 1A). Opacified small bowel presented almost entirely on the right side (Figure 1B). Upper gastrointestinal tract barium studies revealed that the duodenum ran caudally in a straight line from the first part onwards. The fourth duodenal segment and the normal duodeno-jejunal junction (Treitz ligament) were not developed (Figure 2A). Barium enema revealed that all colon segments with the cecum were found to the left of the spine. The cecum lay on the left side of the abdomen and the ileum entered it from the right (Figure 2B).

Based on the diagnosis of malrotation, the patient consented to exploratory laparoscopy. No segmented gangrene of the small intestine was present. Adhesions surrounding the SMA and cecal bands attaching the duodenum and right colon were noted. The Ladd's procedure was performed. In detail, the cecum and right colon were rotated medially to expose the duodenum. The base of the mesentery was widened by incising the peritoneum. Then, the duodenum was moved until it was oriented inferiorly toward the right lower quadrant. The entire length of bowel was examined to assure that no other obstructive bands or kinks were present. The small bowel was then placed on the right side of the abdomen, and the colon was placed on the left side of the abdomen. Finally, the appendix was removed.
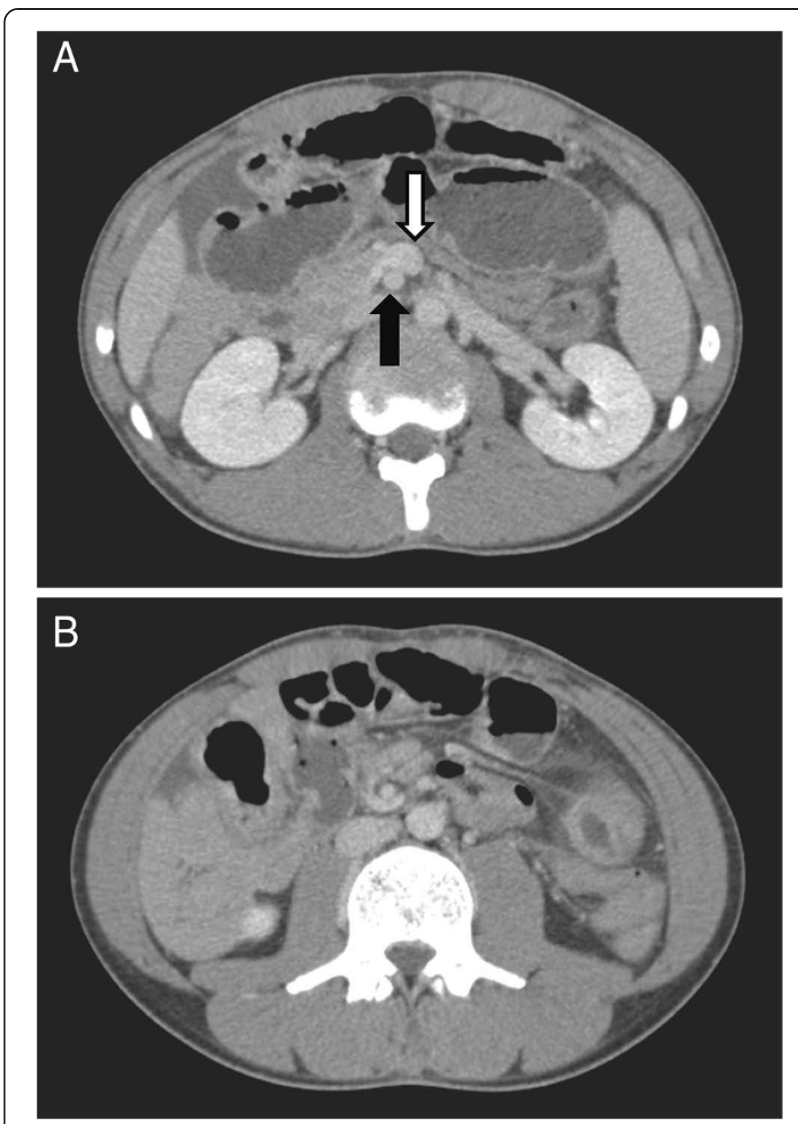

Figure 1 Contrast enhanced CT of the abdomen. A: Contrast enhanced $\mathrm{CT}$ can show the abnormal anatomic location of a right sided small bowel, a left-sided colon, and an abnormal relationship of the superior mesenteric vein (white arrow) situated to the anterior of the superior mesenteric artery (black arrow) instead of to the right. B: Opacified small bowel present almost entirely on the right side.

Operative time was 195 minutes with negligible bleeding. Postoperative course was uneventful. The patient was discharged two days later and has remained asymptomatic without recurrence of abdominal pain three months postoperatively.

\section{Discussion}

Malrotation of the intestinal tract is a congenital anomaly referring to either lack of or incomplete rotation of the fetal intestines around the axis of the superior mesenteric artery during fetal development. The malrotaion of the gut and abnormal location of the cecum produces a narrow superior mesenteric vascular pedicle, as opposed to the normally broadbased small bowel mesentery. This narrow superior mesenteric artery takeoff and lack of posterior peritoneal fusion predispose the patient to subsequent midgut volvulus and obstruction with potential vascular catastrophe. 


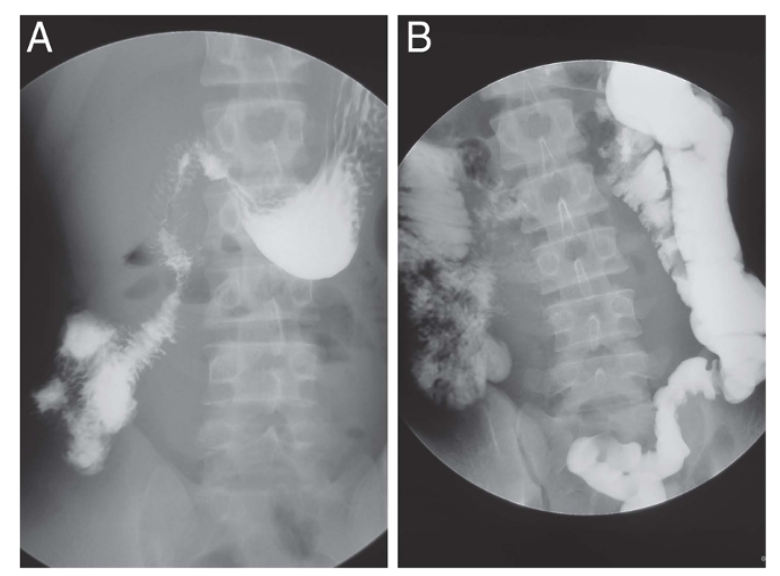

Figure 2 Gastrointestinal contrast studies. A: Upper gastrointestinal contrast studies showed malrotation of the small bowel without evidence of the duodenum crossing the lumbar spine. B: All small bowel was noted to be sequestered on the right side of the abdomen. The cecum lay on the left side of the abdomen and the ileum entered it from the right.

Approximately $85 \%$ of malrotation cases present in the first two weeks of life $[5,6]$. However, presentation of intestinal malrotation is very rare and its incidence has been reported to be between $0.2 \%$ and $0.5 \%$ [7]. True incidence of malrotation in older children or adults is unclear, because a number of patients may be asymptomatic. Not all patients with malrotation present with symptoms. Even once the anomaly is discovered, many live without complaint.

In adults or older children, the difficulty of diagnosis results from both the absence of specific physical findings and the low frequency in adults [8,9]. Midgut malrotation in adults presents in numerous ways and the symptoms are non-specific. There are no typical sets of symptoms that are diagnostic of clinical problems. Symptoms in the adult patient are often mistaken for irritable bowel syndrome, peptic ulcer disease, biliary and pancreatic disease, and psychiatric disorders [8]. The location of the pain may vary from the epigastric region to the left upper abdominal quadrant, and the pain may be described as either intermittent cramping or persistent aching. It most often occurs postprandially and may last several minutes to an hour. Our patient had experienced abdominal distension, nausea, vomiting, and vague abdominal pain several times before, but the symptoms had always disappeared spontaneously.

Frequently, the plain radiograph is normal or may show an incomplete bowel obstruction. Specific findings that are diagnostic of malrotation can be detected through the use of both upper and lower gastrointestinal tract barium studies, angiography of the superior mesenteric artery, CT scan, and often emergency laparotomy.
Occasionally, an abdominal radiograph will show dilated bowel loops with the orientation of a spiral nebula in the midabdomen. Barium studies may reveal a dilated duodenal loop caused by bowel obstruction with a spiral configuration of the proximal jejunal loops. CT is also used to investigate small-bowel volvulus and various signs have been described. Characteristic findings include the positioning of the superior mesenteric vein lying to the left or anterior to the artery because of torsion of the mesentery around its attachment, the presence of a right-sided duodeno-jejunal junction, the absence of a cecal gas shadow on the patient's right side, or third and fourth duodenal junction that does not cross the patient's spine $[10,11]$.

Management of intestinal rotation without midgut volvulus is controversial. In general, symptomatic patients with malrotation should be treated with surgical intervention. The classic treatment for incomplete intestinal rotation is the Ladd procedure, which requires mobilization of the right colon and cecum by division of Ladd bands, mobilization of the duodenum, division of adhesions around the superior mesenteric artery to broaden the mesenteric base, and an appendectomy [12-14]. Spigland et al. recommended that all patients with malrotation are candidates for laparotomy, even if they are asymptomatic [15]. Mozziotti et al. recently reported a series of malrotation patients managed successfully with laparoscopic intervention [16]. Laparoscopy can be used to determine the position of the Treitz ligament and whether the cecum is fixed in the right lower quadrant. If the patient is decided to be at risk for volvulus (i.e. a shortened mesenteric pedicle), a Ladd's procedure can be accomplished laparoscopically with good long-term results $[16,17]$. Due to the abnormal cecal position inflicted by malrotation, patients with associated appendicitis will demonstrate atypical symptoms with pain projected to the left of the middle line since the appendix will not be located in the normal area in the abdomen. This could lead to confusion and delay in diagnosing appendicitis in the future. Therefore, appendectomy is usually performed during surgical intervention.

Although most of the literature consists of occasional case reports or small case series, we searched for literature published between 1983 and 2012 using PubMed and Web Japan Medical Abstracts Society and found 37 reported cases of teenage patients (ages 13 through 19) with intestinal malrotation (Table 1). Twenty patients were male and seventeen were female. The diagnosis could be made by radiographic studies in all these patients. Patients presented with a variety of gastrointestinal disorders. Abdominal pain was the most frequent symptom (30/37). Other symptoms were nausea, feeding intolerance, reflux, and respiratory problems. The Ladd 
Table 1 Reported cases of intestinal malrotaion (13-19 years old)

\begin{tabular}{|c|c|c|c|c|c|c|}
\hline Year & Author & Journal & Age & Gender & Symptoms & Surgery \\
\hline 1991 & Ko, et al. & Jpn J Surg (in Japanese) & 19 & $\mathrm{~F}$ & abdominal distention & Ladd procedure \\
\hline 1992 & Lal, et al. & Indian J Gastroenterol & 17 & $\mathrm{~F}$ & abdominal pain, vomiting & gastrojejunostomy, vagotomy \\
\hline 1994 & Pelucio, et al. & Am J Emerg Med & 15 & M & abdominal pain & Ladd procedure \\
\hline 1997 & Kimura, et al. & Jpn J Clin Surg (in Japanese) & 16 & M & vomiting & Ladd procedure \\
\hline 1997 & Ishida, et al. & J Jpn Soc Pediatr Surg (in Japanese) & 13 & $\mathrm{~F}$ & abdominal pain, vomiting & Ladd procedure \\
\hline 1997 & Yahata, et al. & Surg Laparosc Endosc & 17 & $\mathrm{~F}$ & abdominal pain & laparoscopic Ladd procedure \\
\hline 1998 & Yokota, et al. & $\begin{array}{l}\text { Kesennuma Hosp Medical J } \\
\text { (in Japanese) }\end{array}$ & 15 & $\mathrm{~F}$ & abdominal pain & Ladd procedure \\
\hline 1999 & Kang, et al. & J Jpn Soc Pediatr Surg (in Japanese) & 16 & M & abdominal pain, vomiting & Ladd procedure \\
\hline 1999 & Yamashita, et al. & Surg Endosc & 13 & $\mathrm{~F}$ & vomiting & laparoscopic Ladd procedure \\
\hline 2000 & Walsh, et al. & J Pediatr Surg & 13 & $\mathrm{~F}$ & abdominal pain & laparoscopic Ladd procedure \\
\hline 2001 & Horiba, et al. & J Jpn Clin Surg (in Japanese) & 17 & M & vomiting & Ladd procedure \\
\hline 2003 & Tsumura, et al. & Surg Endosc & 15 & $\mathrm{~F}$ & abdominal pain & laparoscopic Ladd procedure \\
\hline 2003 & Singer, et al. & J Am Coll Surg & 19 & M & abdominal pain, vomiting & Ladd procedure \\
\hline 2004 & Tseng, et al. & JBR-BTR & 14 & $\mathrm{~F}$ & abdominal pain & Ladd procedure \\
\hline 2005 & Sato, et al. & Hokkaido Surg J (in Japanese) & 18 & M & abdominal pain & release of ileus \\
\hline 2005 & Kamiyama, et al. & Radiat Med & 14 & M & abdominal pain & Ladd procedure \\
\hline 2007 & $\begin{array}{l}\text { Vechvitvarakul, } \\
\text { et al. }\end{array}$ & J Pediatr Surg & 13 & M & $\begin{array}{l}\text { abdominal pain, nausea, } \\
\text { vomiting }\end{array}$ & $\begin{array}{l}\text { Ladd procedure, } \\
\text { appendectomy }\end{array}$ \\
\hline 2007 & Kusuda, et al. & $\begin{array}{l}\text { J Abdominal Emergency Medicine } \\
\text { (in Japanese) }\end{array}$ & 17 & M & abdominal pain & Ladd procedure \\
\hline 2007 & Draus, et al. & Am Surg & 17 & $\mathrm{~F}$ & abdominal pain, nausea & laparoscopic Ladd procedure \\
\hline 2008 & Duran, et al. & Turk J Gastroenterol & 17 & $\mathrm{~F}$ & abdominal pain & division of adhesions \\
\hline 2008 & Uchida, et al. & J Pediatr Surg & 13 & $\mathrm{~F}$ & vomiting & Bypass \\
\hline 2009 & Fukushima, et al. & Jpn J Endosc Surg (in Japanese) & 15 & $\mathrm{~F}$ & abdominal pain, distention & laparoscopic Ladd procedure \\
\hline 2009 & Tazaki, et al. & $\begin{array}{l}\text { J Abdominal Emergency Medicine } \\
\text { (in Japanese) }\end{array}$ & 14 & M & abdominal pain, vomiting & release of ileus \\
\hline 2009 & $\begin{array}{l}\text { Shimodaira, } \\
\text { et al. }\end{array}$ & $\begin{array}{l}\text { J of Jpn Soc Psychosomatic Med } \\
\text { (in Japanese) }\end{array}$ & 17 & M & vomiting & laparoscopic Ladd procedure \\
\hline 2009 & Fujii, et al. & J Jpn Clin Surg (in Japanese) & 14 & M & vomiting & Ladd procedure \\
\hline 2009 & Mano, et al. & J Jpn Soc Pediatr Surg (in Japanese) & 18 & M & abdominal pain & laparoscopic Ladd procedure \\
\hline 2010 & Watanabe, et al. & $\begin{array}{l}\text { J Jpn Soc Gastrointestinal Dis (in } \\
\text { Japanese) }\end{array}$ & 19 & $\mathrm{~F}$ & abdominal pain & release of ileus \\
\hline 2010 & Takazawa, et al. & Jpn J Pediatr Surg Nutr (in Japanese) & 14 & M & vomiting, distention & resection of necrotic intestine \\
\hline 2011 & Kokado, et al. & J Jpn Soc Pediatr Surg (in Japanese) & 13 & $\mathrm{~F}$ & abdominal pain, vomiting & fixation of colon \\
\hline 2011 & Lam, et al. & J Pediatr Surg & 14 & M & abdominal pain, vomiting & resection of necrotic intestine \\
\hline 2012 & Nath, et al. & Ann R Coll Engl & 16 & M & abdominal pain & laparoscopic Ladd procedure \\
\hline 2012 & Jain, et al. & Case Rep Radiol & 15 & M & abdominal pain & Ladd procedure \\
\hline 2012 & Wanjari, et al. & N Am J Med Sci & 17 & M & abdominal pain, vomiting & laparoscopic Ladd procedure \\
\hline 2012 & Macedo, et al. & Einstein & 13 & $\mathrm{~F}$ & abdominal pain & laparoscopic Ladd procedure \\
\hline 2012 & Tran, et al. & J Pediatr Surg & 18 & M & abdominal pain & Ladd procedure \\
\hline 2012 & Katsura, et al. & J Jpn Clin Surg (in Japanese) & 19 & $\mathrm{~F}$ & abdominal pain & resection of necrotic intestine \\
\hline 2013 & Nakajima, et al. & present case & 17 & M & abdominal pain, vomiting & laparoscopic Ladd procedure \\
\hline
\end{tabular}


procedure was performed on 27 patients; on 12 patients the procedure was conducted laparoscopically.

An important point is that since many patients with intestinal malrotation are asymptomatic, everyone in the medical community should be made aware of the problem. Also, patients with acute volvulus should be treated promptly. Some asymptomatic adults may not need surgery. Of note, there is always the possibility that laparoscopic surgery will not entirely rule out the chance of acute volvulus; it could introduce problems such as band adhesion and future adhesive small bowel obstruction.

In conclusion, a number of teenage patients with intestinal malrotation present with symptoms. Increased awareness of this condition and an understanding of its varied presentation at different ages may reduce the time needed to diagnose the problem and improve patient outcome. Laparoscopy is an excellent technique for the evaluation and definitive management of patients without midgut volvulus with intestinal rotation abnormalities.

\section{Consent}

Written informed consent was obtained from the patient's guardian/parent/next in keen for publication of this report and any accompanying images. A copy of the written consent is available for review by the Editor-in -Chief of this journal.

\section{Competing interests}

The authors declare that they have no competing interests.

\section{Authors' contribution}

YN, HS, NY, TY, TO and MT were involved in preoperative diagnosis and postoperative care. NM conceived performed the literature search. TY, RS, SN, TS and HO performed the operation, involved in the preoperative and postoperative care. AN and JK conceived the write up, performed the literature search and drafted the manuscript. All authors read and approved the manuscript for submission.

\section{Author details \\ ${ }^{1}$ Department of Emergency, Disaster and Critical Care Medicine, Hyogo College of Medicine, 1-1 Mukogawa, Nishinomiya, Hyogo 663 8501, Japan. ${ }^{2}$ Department of Pediatric Surgery, Hyogo College of Medicine, Nishinomiya, Hyogo, Japan.}

Received: 22 February 2013 Accepted: 12 May 2013

Published: 17 May 2013

\section{References}

1. Maxson RT, Franklin PA, Wagner CW: Malrotation in the older child: surgical management, treatment, and outcome. Am Surg 1995, 61:135-138.

2. Yanez R, Spitz L: Intestinal malrotation presenting outside the neonatal period. Arch Dis Child 1986, 61:682-685.

3. Hsu SD, Yu JC, Chou SJ, Hsieh HF, Chang TH, Liu YC: Midgut volvulus in an adult with congenital malrotation. Am J Surg 2008, 195:705-707.

4. Wanjari AK, Deshmukh AJ, Tayde PS, Lonkar Y: Midgut malrotation with chronic abdominal pain. N Am J Med Sci 2012, 4:196-198.

5. Gamblin TC, Stephens RE Jr, Johnson RK, Rothwell M: Adult malrotation: a case report and review of the literature. Curr Surg 2003, 60:517-520.

6. Ford EG, Senac MO Jr, Srikanth MS, Weitzman JJ: Malrotation of the intestine in children. Ann Surg 1992, 215:172-178.

7. Wang CA, Welch CE: Anomalies of intestinal rotation in adolescents and adults. Surgery 1963, 54:839-855.
8. Fukuya T, Brown BP, Lu CC: Midgut volvulus as a complication of intestinal malrotation in adults. Dig Dis Sci 1993, 38:438-444.

9. Nehra D, Goldstein AM: Intestinal malrotation: varied clinical presentation from infancy through adulthood. Surgery 2011, 149:386-393.

10. Nichols DM, Li DK: Superior mesenteric vein rotation: a CT sign of midgut malrotation. AJR Am J Roentgenol 1983, 141:707-708.

11. Singh S, Das A, Chawla AS, Arya SV, Chaggar J: A rare presentation of midgut malrotation as an acute intestinal obstruction in an adult: Two case reports and literature review. Int J Surg Case Rep 2013, 4:72-75.

12. Schultz $L R$, Lasher $E P$, Bill AH Jr: Abnormalities of rotation of the bowel. Am J Surg 1961, 101:128-133.

13. Matzke GM, Moir CR, Dozois EJ: Laparoscopic ladd procedure for adult malrotation of the midgut with cocoon deformity: report of a case. J Laparoendosc Adv Surg Tech A 2003, 13:327-329.

14. Badea R, Al Hajjar N, Andreica V, Procopet B, Caraiani C, Tamas-Szora A: Appendicitis associated with intestinal malrotation: imaging diagnosis features. Case report. Med Ultrason 2012, 14:164-167.

15. Spigland N, Brandt ML, Yazbeck S: Malrotation presenting beyond the neonatal period. J Pediatr Surg 1990, 25:1139-1142.

16. Mazziotti MV, Strasberg SM, Langer JC: Intestinal rotation abnormalities without volvulus: the role of laparoscopy. J Am Coll Surg 1997, 185:172-176.

17. Waldhausen JH, Sawin RS: Laparoscopic Ladd's procedure and assessment of malrotation. J Laparoendosc Surg 1996, 6(Suppl 1):S103-S105.

doi:10.1186/1749-7922-8-19

Cite this article as: Nakajima et al: Successful treatment of a 14-year-old patient with intestinal malrotation with laparoscopic Ladd procedure: case report and literature review. World Journal of Emergency Surgery 2013 8:19.

\section{Submit your next manuscript to BioMed Central and take full advantage of:}

- Convenient online submission

- Thorough peer review

- No space constraints or color figure charges

- Immediate publication on acceptance

- Inclusion in PubMed, CAS, Scopus and Google Scholar

- Research which is freely available for redistribution 